\title{
Acquired aplastic anaemia: still a serious disease
}

\author{
D K H Webb, I M Hann, J M Chessells
}

\begin{abstract}
Over 15 years, 42 children aged 2-14 years were diagnosed as having acquired aplastic anaemia. Adequate clinical details were available for 38 children who were categorised as very severe $(n=13)$, severe $(n=16)$, or nonsevere $(n=9)$ by the modified Camitta criteria.

Treatment varied over the study period. Seven children received a bone marrow allograft from a full match family donor and three a matched unrelated donor transplant after failed treatment with antilymphocyte globulin. The remainder were treated with antilymphocyte globulin $(n=11)$, antilymphocyte globulin and oxymetholone $(n=4)$, oxymetholone with or without prednisolone $(n=12)$, or supportive treatment alone $(n=1)$.

With a minimum follow up of one year since treatment, the five year survival was $70 \%$ for bone marrow transplantation with a family donor, $\mathbf{3 0} \%$ for antilymphocyte globulin, and $25 \%$ for oxymetholone. All three children with a matched unrelated donor transplant died.

The prognosis of acquired aplastic anaemia remains poor for most children and new approaches to treatment are urgently required.
\end{abstract}

Aplastic anaemia is a rare but often fatal disorder of haemopoiesis. It is characterised by blood pancytopenia and bone marrow hypoplasia and affected 2-4 per million children under 15 years in one Danish series. ${ }^{1}$ The incidence is higher in the Orient, ${ }^{2}$ probably reflecting increased exposure to environmental toxins and hepatitis. ${ }^{3}$ The bone marrow failure may be constitutional or acquired: constitutional causes include Fanconi's anaemia, dyskeratosis congenita, and Shwachman's syndrome. Although drugs, toxins, and viruses have been implicated in the aetiology of acquired aplastic anaemia, over $70 \%$ of cases are idiopathic. ${ }^{4}$ There is conflicting evidence that in some cases acquired aplastic anaemia is an immune mediated disorder, ${ }^{56}$ and $50 \%$ of patients show an improvement with immune suppression. ${ }^{7}$ Only $20 \%$ of severely affected children survive one year from diagnosis with supportive care alone, ${ }^{8}$ and although allogeneic bone marrow transplantation may be curative, only $30 \%$ of patients have a suitable family donor. There are few paediatric data, and the outcome for children treated over a 15 year period at a single institution is presented.

\section{Patients and methods}

Over the 15 year period, January 1974 to
December 1988, 42 children were diagnosed as having acquired aplastic anaemia at the Hospital for Sick Children, Great Ormond Street, London. The medical records were reviewed for details of age, sex, case history, laboratory investigations, aetiology, and outcome. Adequate details were available for 38 children (17 boys, 21 girls) with a median age of 6 years (range 2-14) at diagnosis.

Laboratory investigations included the acidified serum lysis (Ham's) test for paroxysmal nocturnal haemoglobinuria, cytogenetic analysis of blood lymphocytes stressed with diepoxybutane or mitomycin C for Fanconi's anaemia, virology, liver function tests, and a trephine biopsy to assess accurately marrow cellularity. Severity was assessed by the modified Camitta criteria. ${ }^{9}$ Children with severe aplastic anaemia had a pancytopenia with at least two of the following features: neutrophil count $<0.5 \times 10^{9} / 1$, platelet count $<20 \times 10^{9} / 1$, corrected reticulocyte count $<1 \%$, and a bone marrow trephine with residual haemopoietic cells $<30 \%$ of normal cellularity. Children with very severe aplastic anaemia fulfilled these criteria, but had a neutrophil count of $<0 \cdot 2 \times 10^{9} / 1$, whereas more mildly affected individuals had non-severe aplastic anaemia.

Treatment varied over the study period but all children received broad spectrum antibiotics, antifungal therapy, and blood and platelet transfusions as clinically indicated. Families were tissue typed soon after diagnosis. Children with severe or very severe aplastic anaemia and an HLA full match family doctor were treated by allogeneic bone marrow transplantation, either at Great Ormond Street, the Royal Marsden, or Hammersmith Hospitals. Pretransplant conditioning was with cyclophosphamide in each case, and graft versus host disease prophylaxis was by methotrexate $(n=5)$, cyclosporin $(n=4)$, or T cell depletion $(n=1)$. Before 1980 children without a bone marrow donor, or with non-severe disease, were treated by oral oxymetholone $2.5 \mathrm{mg} / \mathrm{kg} /$ day usually combined with prednisolone. Since 1980 these children received horse antilymphocyte globulin (ALG, Merieux) 1.5 vials $/ 10 \mathrm{~kg} /$ day for five days by a central venous catheter, with prophylaxis of serum sickness by methylprednisolone $5 \mathrm{mg} / \mathrm{kg} /$ day. Children treated with supplementary androgens received oxymetholone $2.5 \mathrm{mg} / \mathrm{kg} /$ day for four months after completion of antilymphocyte globulin. If there was an inadequate response to horse antilymphocyte globulin by four months after treatment patients received a five day course of rabbit antilymphocyte globulin. 
A complete response to treatment was defined as a normal blood count for the child's age and sex, a partial response as a lesser degree of improvement with independence from blood and platelet transfusion, and no response as failure to improve with treatment. Survival curves for each treatment group were estimated by the method of Kaplin and Meier.

\section{Results}

At diagnosis there were 13 children with very severe, 16 with severe, and nine with non-severe aplastic anaemia. All had bruising and pallor but only six had frank bleeding caused by epistaxis $(n=1)$, gingival/buccal bleeding $(n=2)$, melaena $(n=2)$, and haematemesis $(n=1)$. Haematological findings at presentation are shown in table 1. Three children had a history or serological evidence of recent infection (varicella, non- $A$, non-B hepatitis, and mycoplasma) but the aetiology was unknown in the other cases.

One child died of bleeding and sepsis three weeks after diagnosis and before any specific treatment.

\section{OXYMETHOLONE ALONE}

Twelve children were treated with oxymetholone (table 2). Two children had a complete and two a partial response, but one child with a partial response subsequently deteriorated and died. There were three survivors: two at six years and one at seven years from diagnosis. Two patients were subsequently lost to follow up, and the remaining child had a mild persistent neutropenia.

IMMUNE SUPPRESSION

Eighteen children were treated by immune suppression: 11 received antilymphocyte globulin alone and seven antilymphocyte globulin followed by oxymetholone. A response was observed in nine cases: four had a complete response, but

Table 1 Haematological findings at presentation

\begin{tabular}{llll}
\hline & $\begin{array}{l}\text { No of } \\
\text { children }\end{array}$ & Median & Range \\
\hline Haemoglobin $(\mathrm{g} / \mathrm{l})$ & 38 & 74 & $30-110$ \\
Neutrophil count $\left(\times 10^{9} / \mathrm{l}\right)$ & 38 & $0 \cdot 4$ & $0 \cdot 1-2$ \\
Platelet count $\left(\times 10^{9} / \mathrm{l}\right)$ & 38 & 11 & $1-40$ \\
Corrected reticulocyte count $(\%)$ & 27 & $0 \cdot 4$ & $0 \cdot 1-2 \cdot 5$ \\
\hline
\end{tabular}

of five children with a partial response, four deteriorated and died. Nine children had no response: eight died but one child survives after a partial response to treatment with cyclosporin. Of 10 children who received a second course of antilymphocyte globulin, one had a complete response, two a partial response, and one of these is a long term survivor with persistent neutropenia and thrombocytopenia. Patient numbers were too small to allow analysis for the effects of age, sex, severity, and oxymetholone on response to antilymphocyte globulin.

\section{BONE MARROW TRANSPLANTATION}

Ten children had an allogeneic bone marrow transplant: seven from an HLA full match family donor and three from a matched unrelated donor after treatment with antilymphocyte globulin failed. All three children with a matched unrelated donor failed to engraft and died. Two children who failed to engraft after an initial transplant from a family donor were successfully treated after preparation with cyclophosphamide and antilymphocyte globulin. Two children died of varicella two months and three years after bone marrow transplantation. Five survive in normal health two, four, 12, 13, and 13 years from transplantation. Mild acute graft versus host disease affected one child, but no surviving child had chronic graft versus host disease.

The five year actuarial survival for bone marrow transplantation with a matched family donor was $70 \%$, for immune suppression by antilymphocyte globulin $30 \%$, and for androgen treatment alone $25 \%$ (figure). The survival advantage for children treated by family donor

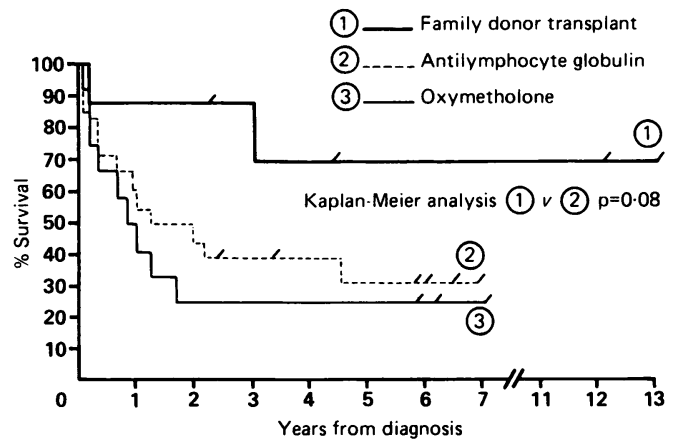

Outcome for children with aplastic anaemia subdivided by treatment group.

Table 2 Characteristics of children with aplastic anaemia by treatment group

\begin{tabular}{|c|c|c|c|}
\hline & $\begin{array}{l}\text { Oxymetholone } \\
(n=12)\end{array}$ & $\begin{array}{l}\text { Antilyphocyte } \\
\text { globulin } \\
(n=18)\end{array}$ & $\begin{array}{l}\text { Bone marrow } \\
\text { transplantationt } \\
(n=7)\end{array}$ \\
\hline $\begin{array}{l}\text { Median age in years (range) } \\
\text { Sex M:F } \\
\text { Disease severity (non-severe:severe:very severe) } \ddagger \\
\text { Haemoglobin }(\mathrm{g} / \mathrm{l}) \S \\
\text { Reticulocytes }(\%) \\
\text { Neutrophil count }\left(\times 10^{9} / \mathrm{l}\right) \S \\
\text { Platelet count }\left(\times 10^{9} / 1\right) \S \\
\text { No who survived }\end{array}$ & $\begin{array}{l}5(3-10) \\
7: 5 \\
5: 2: 5 \\
70 \\
1 \\
0 \cdot 4 \\
15 \\
3\end{array}$ & $\begin{array}{l}8(2-14) \\
5: 13 \\
3: 10: 5 \\
70 \\
0 \cdot 2 \\
0 \cdot 3 \\
11 \\
6\end{array}$ & $\begin{array}{l}7(1-11) \\
4: 3 \\
1: 4: 2 \\
70 \\
0 \cdot 2 \\
0 \cdot 5 \\
11 \\
5\end{array}$ \\
\hline
\end{tabular}

One child received supportive treatment only.

†Allogeneic bone marrow transplantation from a family donor

$\neq$ Disease severity assessed by modified Camitta criteria.

SAll haematology indices are median values. 
transplant was not significant on Kaplan-Meier analysis $(\mathrm{p}=0.08)$.

\section{Discussion}

Acquired aplastic anaemia remains a rare but severe disease in childhood. Most cases are idiopathic - in this series only three of 34 children had a possible causative agent, each being infective-and review of the literature indicates that drug or toxin induced aplasia and paroxysmal nocturnal haemoglobinuria are rare among Western children. A higher incidence of aplastic anaemia due to drug toxicity and hepatitis has been described in the Orient. ${ }^{23}$ The mechanism of bone marrow failure is unclear, although there is conflicting evidence from culture experiments for an immune mediated suppression of haemopoiesis. There have been reports of $40-70 \%$ of children ${ }^{81011}$ and $50-60 \%$ of adults ${ }^{7}$ showing an improvement after immune suppressive treatment with antilymphocyte globulin, although blood values return to normal in only a minority of cases. In several series up to $50 \%$ of adults treated with antilymphocyte globulin have subsequently developed a clonal disorder of haemopoiesis including acute myeloid leukaemia, paroxysmal nocturnal haemoglobinuria, or myelodysplasia. ${ }^{1213}$ This argues for an underlying defect of haemopoiesis in many cases. These complications have not been described in children, however, and it is possible that a different mechanism of bone marrow failure operates in childhood. Despite a male preponderance in the adult series, there was no sex difference in incidence in this study.

In this series 29 of 38 children had severe or very severe aplastic anaemia. An increased severity of aplastic anaemia in childhood has been described, ${ }^{14}$ although the small number of paediatric cases precludes definite conclusions on relative severity. Haemorrhage or sepsis at diagnosis is associated with a poor prognosis, ${ }^{15}$ and in our report five of six children with bleeding at presentation died.

Allogeneic bone marrow transplantation from an HLA full match family donor is the treatment of choice for severe and very severe aplastic anaemia, and in our series five of seven children are alive in full health; this is consistent with other reports. ${ }^{811} 1617$ It is important that all families are tissue typed soon after diagnosis, and children with a donor and severe or very severe aplastic anaemia should be treated by early transplantation. Bone marrow transplantation from a matched unrelated donor or a family donor with minor mismatch has been successful in some cases. ${ }^{18}{ }^{19}$ Although three children with a matched unrelated donor in this series died, all were late transplants in pretreated patients with a high risk of treatment failure. ${ }^{20}$ Our present policy is to initiate a matched unrelated donor search for all severely affected children without a suitable family donor soon after diagnosis as the published results of matched unrelated donor transplants are more promising than the outcome with other second line treatment. Preparative regimens influence survival and there have been poor results in patients treated by schedules including methotrexate. The combination of cyclophosphamide with cyclosporin carries a low risk of graft rejection $(17 \%)^{21}$ and graft versus host disease, although the incidence of long term sequelae such as infertility and secondary malignancies is unclear.

The survival after treatment with oxymetholone alone was poor, consistent with other series, ${ }^{15}$ and no better than that obtained with supportive care. ${ }^{14}$ Antilymphocyte globulin was of only limited benefit in this series, with no evidence of a survival advantage compared with androgen treatment. Only five of 18 children showed a complete response and patients with a partial response had a high relapse rate. These data are consistent with the $40-50 \%$ five year survival reported for children with severe aplastic anaemia receiving antilymphocyte globulin in four paediatric series. ${ }^{811} 1720$ Werner et al described a $70 \%$ response rate but numbers were small and survival data limited in their series. ${ }^{10} \mathrm{~A}$ higher response and survival rate has been described in predominantly adult patients with non-severe aplastic anaemia, ${ }^{7}$ and a poor outcome is predicted by young age, female sex, neutrophil count $<0.2 \times 10^{9} / 1$, and bleeding or sepsis at diagnosis. ${ }^{15}$ The approach to treatment for a child with non-severe aplastic anaemia and an HLA full match family donor is controversial. It is probably best managed by a trial of antilymphocyte globulin in the first instance and with bone marrow transplantation reserved for children with other, poor prognostic features, or who have less than a complete response to antilymphocyte globulin.

For children who fail first line treatment responses have been described after immune suppression by cyclosporin or high doses of methylprednisolone. ${ }^{22}{ }^{23}$ Of these cyclosporin is the more promising with a $30 \%$ response rate in patients who relapse after or fail to respond to antilymphocyte globulin, and in our series one child has shown steady improvement without toxicity. Treatment with recombinant granulocyte macrophage colony stimulating factor is effective in raising the neutrophil count in aplastic anaemia, but had no effect in patients with very low neutrophil counts or sepsis at the time of treatment, ${ }^{24}$ and it is essentially supportive therapy.

Despite advances in treatment the prognosis of aplastic anaemia remains poor in a large proportion of cases. New approaches to treatment are required to reduce the morbidity and mortality associated with this disease.

We wish to thank Dr JM Hows, Professor EC Gordon-Smith, the late Professor TS McElwain, and Dr Ray Powles for their help in the management of these patients, and Miss Sonia Taylor who typed this manuscript.

1 Clausen N. A population study of severe aplastic anaemia in children. Acta Paediatr Scand 1986;75:58-63.

Young NS, Issara Grasil S, Chick CW, Takaku F. Aplastic anaemia in the Orient. Br f Haematol 1986;62:1-6.

3 Liang DC, Lin KH, Lin DT, Yang CP, Hung KL, Lin KS Post-hepatitic aplastic anaemia in children in Taiwan, hepatitis prevalent area. Br f Haematol 1990;74:487-91.

4 Alter BP, Potter NU, Li FP. Classification of the aplastic anaemias. Clin Haematol 1978;7:431-65.

Hoffman $R$ Zaniani ED, Lutton JD Suppression of erytran R, Zanjani ED, Lutton JD. Suppression of eryhroid colony formation by lymphocytes from patients with aplastic anemia. N Engl f Med 1977;296:10-3.

6 Sullivan R, Quesenberry PJ, Parkman R, et al. Aplastic anemia: lack of inhibitory effect of bone marrow lymphocytes on in vitro granulopoiesis. Blood 1980;56:625-32. 7 Marsh JCW, Hows JM, Bryett KA, et al. Survival after anti- 
lymphocyte globulin therapy for aplastic anemia depends 1987:70:1046-52.

8 Halpérin DS, Grisaru D, Freedman MH, Saunders EF Severe acquired aplastic anemia in children: 11 year experience with bone marrow transplantation and immunosuppressive therapy. Am $\mathcal{F}$ Pediatr Hematol Oncol 1989;11: $304-9$.

9 Camitta B, O'Reilly RJ, Sensenbrenner L, et al. Antithoracic duct lymphocyte globulin therapy of severe aplastic anemia. Blood 1983;62:883-5.

10 Werner EJ, Stout RD, Valdez LP, Harris RE. Immunosuppressive therapy versus bone marrow transplantation for children with aplastic anemia. Pediatrics 1989;83:61-4

11 Locasciulli A, Van't Veer L, Bacigalupo A, et al. Treatment with marrow transplantation or immunosuppression of childhood acquired severe a the EBMT SAA Working Party. Bone Marrow Transplantation 1990;6:211-7.

12 Tichelli A, Greatcooke A, Wursch A, et al. Late haematological complications in severe aplastic anaemia. $B r$ f Haematol 1988;69:413-8.

13 De Planque MM, Kluin-Nelemans HC, Van Krieken HJM, et al. Evolution of acquired severe aplastic anaemia to myelodysplasia and subsequent leukaemia in adults. $B r$ 7 Haematol 1988;70:55-62.

14 Najean Y, Girot R, Baumelou E. Prognostic factors and evolution of acquired aplastic anemia in childhood. $A m$ f Pediatr Hematol Oncol 1984;4:273-83.

15 Bacigalupo A. Treatment of severe aplastic anaemia. In: Gordon-Smith EC, ed. Clinical haematology. London: Baillière Tindall, 1989.

16 McGlare PB, Haake R, Miller W, Kim T, Kersey J, Ramsey NK. Therapy of severe aplastic anemia in young adults and children with allogeneic bone marrow transplantation. Blood 1987;70:1325-30.

17 Bayerer E, Champlin R, Ho W, et al. Comparison between bone marrow transplantation and antilymphocyte globulin in the treatment of young patients with severe aplastic anemia. I Pediatr 1984;105:920-5.

18 Bacigalupo A, Hows JM, Gordon-Smith EC, et al. Bone marrow transplantation for severe aplastic anaemia from donors other than HLA identical siblings: a report of the BMT Working Party. Bone Marrow Transplantation 1988;3: 531.

19 Hows JM, Yin JL, Marsh JCW, et al. Histocompatible unrelated volunteer donors compared with HLA nonidentical donors in marrow transplantation for aplastic anemia and leukemia. Blood 1986;68:1322.

20 Locasciulli A, Porta F, Vossen JM, Bacigalupo A. Treatment of acquired severe aplastic anaemia (SAA) in children: an analysis of the EBMT-SAA Working Party. Bone Marrow analysis of the EBMT-SAA Workin

21 Hows JM, Gluckman E, Devergie A, Bacigalupo E. The effect of conditioning protocols on outcome of BMT for severe aplastic acquired anaemia. Bone Marrow Transplantation 1989;4(suppl 2):92.

22 Leonard EM, Raefsky E, Griffith P, et al. Cyclosporin therapy in aplastic anaemia, congenital and acquired red cell aplasia. $\mathrm{Br} \mathcal{J}$ Haematol 1989;72:278-84.

23 Ozsöylu S, Coskun T, Minassezi S. High dose intravenous glucocorticoids in the treatment of childhood acquired aplastic anaemia. Scand f Haematol 1984;33:309-16.

24 Raghavachar A, Frickhofen N, Kaltwasser JP, et al. GM-CSF therapy in aplastic anaemia patients with very severe neutropenia. Bone Marrow Transplantation 1990;5(suppl 2):

Autoantibodies in juvenile chronic arthritis

Autoantibodies against nuclear and other antigens are found in patients with juvenile chronic arthritis. In diseases such as systemic lupus erythematosus and progressive systemic sclerosis family members frequently also have autoantibodies but this aspect has not been investigated as regards juvenile chronic arthritis until now. Such a study has recently been reported from Australia (Southwood et al, Annals of the Rheumatic Diseases 1990; 49:968-72).

They investigated 23 children with the disease and 66 of their first degree relatives. Antinuclear antibodies were found in 16 patients $(70 \%)$ and nine relatives (14\%). In Australia $3.5 \%$ of the general population are thought to have such antibodies. Eighteen out of 20 families had at least one member with autoantibodies in addition to the one with juvenile chronic arthritis. Immunoblotting showed patterns shared between probands and other members in five families.

This study confirms that antinuclear antibodies are common in juvenile chronic arthritis and shows that apparently unaffected family members may also have these antibodies. It seems likely that there is a genetically determined tendency to produce autoantibodies in this as in other autoimmune diseases. 